\title{
Combined bipolar radiofrequency surgery of the tongue base and uvulopalatopharyngoplasty for obstructive sleep apnea
}

\author{
Jan Plzak $k^{1,2}$, Michal Zabrodsky ${ }^{1}$, Jan Kastner ${ }^{1}$, Jaroslav Betka ${ }^{1}$, Jan Klozar ${ }^{1}$
}

1Department of Otorhinolaryngology and Head and Neck Surgery, $1^{\text {st }}$ Faculty
of Medicine, Charles University, Faculty Hospital Motol, Prague, Czech Republic
2Institute of Anatomy, $1^{\text {st }}$ Faculty of Medicine, Charles University, Prague, Czech
Republic

Submitted: 11 February 2011

Accepted: 7 July 2011

Arch Med Sci 2013; 9, 6: 1097-1101

DOI: 10.5114/aoms.2013.39226

Copyright $\odot 2013$ Termedia \& Banach

\section{Abstract}

Introduction: The aim of the study was to investigate the effectiveness of combined bipolar radiofrequency surgery of the tongue base (RFBT) and uvulopalatopharyngoplasty (UPPP) in a single session for obstructive sleep apnea and whether this combination is safe and well tolerated.

Material and methods: Seventy-nine patients with obstructive sleep apnea and both palatal and retroglossal obstruction underwent UPPP with bipolar RFBT. The control group consisted of 35 patients treated by UPPP alone.

Results: The apnea-hypopnea index significantly decreased from 28.7 to 14.1 . The oxygen desaturation index decreased from 15.1 to 10.3. Mean oxygen saturation was unchanged. Subjectively, the Epworth Sleepiness Scale was significantly improved from 10.6 to 7.3 , and the snoring level decreased from 8.4 to 6.0. The overall treatment success rate increased from $41.9 \%$ for UPPP alone to $51.7 \%$ for UPPP + RFBT. No serious adverse events occurred. Two patients had postoperative bleeding from the tonsillar bed after UPPP. Four patients had ulceration of the base of the tongue after RFBT with spontaneous cure. One patient had a taste change in half of the tongue that resolved within two months.

Conclusions: Combined bipolar RFBT and UPPP in a single session is well tolerated and safe surgery in the treatment of obstructive sleep apnea. It is effective in reducing respiratory parameters and subjective symptoms of obstructive sleep apnea. Further advantages are a single session, simple feasibility, bipolar technique and short time of the procedure.

Key words: radiofrequency, obstructive sleep apnea, uvulopalatopharyngoplasty.

\section{Introduction}

After the introduction of uvulopalatopharyngoplasty (UPPP) by Ikematsu in 1963 and its modification by Fujita in 1981 this technique became increasingly widespread in the field of sleep medicine [1]. Although it presented a fundamental expansion of the therapeutic spectrum and introduction of otorhinolaryngologists into sleep medicine, UPPP was merely a modification of well-established surgical procedures in the oropharynx. Uvulopalatopharyngoplasty is nowadays the most frequent surgery used in the treatment of velopharyngeal obstruction in obstructive sleep apnea (OSA). However, an extensive category is formed by OSA patients with a combination of palatal and retroglossal obstruction. In the treatment of

\author{
Corresponding author: \\ Jan Plzak MD, PhD \\ Department \\ of Otorhinolaryngology \\ and Head and Neck Surgery \\ $1^{\text {st }}$ Faculty of Medicine \\ Charles University \\ University Hospital Motol \\ V Uvalu 84 \\ 15006 Prague 5 \\ Czech Republic \\ Phone: +420 224434301 \\ Fax: +420 224434319 \\ E-mail: jan.plzak@lf1.cuni.cz
}


lower pharyngeal obstruction several surgical techniques such as hyoid suspension, genioglossus advancement, and maxillomandibular advancement have been used. Especially maxillomandibular advancement can be very effective. But these procedures are accompanied by significant postoperative morbidity and require rather a long time of hospitalization. Thus they are mostly reserved for second-level surgical treatment or for severe OSA. Radiofrequency thermotherapy of the base of the tongue (RFBT) was first described by Powel in 1999 as minimally invasive surgery in treatment of retroglossal obstruction in OSA [2]. Radiofrequency thermotherapy of the base of the tongue has been proved to be a safe and effective method used for upper and lower pharyngeal obstruction [3, 4]. However, the authors usually reported that several sessions were needed for tongue base reduction. For combination of palatal and retroglossal obstruction in mild and moderate OSA combined RFBT and UPPP seems to be a very useful option of surgical treatment. However, very little has been published about its results so far [5-7].

In this study, we present our results of combined RFBT and UPPP as a single-session procedure for mild and moderate OSA with both palatal and retroglossal obstruction. We investigate the effectiveness of the surgery and whether this combination is safe and well tolerated. We compare the effect of UPPP alone from the period when RFBT was not available at our department to the effect of UPPP + RFBT.

\section{Material and methods}

Seventy-nine patients with OSA and both palatal and retroglossal obstruction underwent UPPP with RFBT between November 2006 and April 2010. As a control group we used 35 patients with OSA and both palatal and retroglossal obstruction who underwent UPPP only between November 2004 and November 2006 when RFBT was not available at our department. Exclusion criteria were history of previous surgery for OSA and only one level of the airway obstruction. All medical procedures were approved by the Institutional Review Board of the hospital.

All the patients underwent preoperatively and 6 months postoperatively complex examination, which included the following items:

\section{Sleep study}

All-night sleep study was performed at the hospital using a Breas Sc20 device (Medicon, Sweden). Nasal airflow was measured by a nasal pressure sensor, thoracic and abdominal movements by inductive plethysmography, snoring was monitored by a microphone, and pulse oximetry was used to monitor oxygen saturation and heart rate. Apneas were defined as cessation of airflow for more than $10 \mathrm{~s}$. Hypopneas were defined as a decrease of $30 \%$ or more of airflow associated with greater than $4 \%$ decline in oxygen saturation. The oxygen desaturation index (ODI) was calculated as the mean number of desaturations of more than $4 \%$ per hour. The apnea-hypopnea index (AHI) was calculated as the total sum of apneas plus hypopneas per hour of sleep. The apnea index (Al) was calculated as the total sum of apneas per hour of sleep.

\section{Physical examination}

Full general ear, nose and throat examination with emphasis on obstruction of the upper aerodigestive apparatus was performed in all the patients. The body mass index (BMI) was calculated by dividing weight $(\mathrm{kg})$ by square of height $\left(\mathrm{m}^{2}\right)$. Friedman staging was used for evaluation of oropharyngeal obstruction - tonsil size was graded 0-4, palatal position was graded $1-4$. This study included only patients in Friedman stage II (tonsil size 0, 1 or 2 and palate position 1 or 2 ; tonsil size 3 or 4 and palatal position 3 or 4) and stage III (tonsil size 0,1 or 2 and palate position 3 or 4) [8]. Cephalometry was performed with measurement of posterior airway space (PAS), the minimal distance between the base of the tongue and the posterior pharyngeal wall, and SNB, the angle formed by the intersection of lines drawn from the sella turcica to the nasion and the nasion to the supramentale. Fibreoptic nasopharyngoscopy was performed during sedation with midazolam to identify the level of obstruction.

\section{Questionnaire}

Snoring was subjectively evaluated using a visual analogue scale (VAS) ranging from 0 (no snoring) to 10 (bed-partner leaves the room). Daytime sleepiness was evaluated by the Epworth Sleepiness Scale (ESS).

\section{Surgical techniques}

Uvulopalatopharyngoplasty under general anesthesia with nasotracheal intubation was carried out in a similar manner to Fujita's technique [1]. A tonsillectomy was performed at first. The uvula was excised and the anterior and posterior palatal pillar was trimmed and reoriented.

Radiofrequency thermotherapy of the base of the tongue was performed using a CelonLab ENT (Olympus, Germany) unit with bipolar electrode probe CelonProSleep Plus with power setting of $7 \mathrm{~W}$. Nine application sites were selected at the dorsal surface of the base of the tongue. Three lesions were in the midline of the base of the tongue: the first one in the foramen caecum region, the second 
$1 \mathrm{~cm}$ before and the third $1 \mathrm{~cm}$ behind the first lesion. The next three lesions were situated $1 \mathrm{~cm}$ right from the first three ones and the last three lesions on the left side (Figure 1). All procedures were performed as a single session, without any additional RFBT under local or general anesthesia later on. All the patients received postoperative antibiotics (cephalosporin) for 5 days. Patients were discharged around three days postoperatively.

Treatment success was defined as more than $50 \%$ reduction of the $\mathrm{AHI}$ and $\mathrm{AHI}$ below 20 .

\section{Statistical analysis}

Statistica 6.0 software (StatSoft, Czech Republic) was run in all statistical analyses. Pre- and postoperative parameters were compared by paired $t$-test. Differences between the 2 patient groups were evaluated by paired $t$-test and $\chi^{2}$ test.

\section{Results}

From the 79 patients of the study group there were 62 males and 17 females; median age 50.5 years; age range $29-62$ years. The control group consisted of 35 patients, 29 males and 6 females; median age 47.9 years; age range 33-59 years. There were no significant differences between the study and control groups in age, gender, body mass index (BMI), preoperative $\mathrm{AHI}, \mathrm{Al}, \mathrm{ODI}$, mean $\mathrm{SpO}_{2}$, ESS, and VAS snoring (Table I). Comparison of preoperative and postoperative data is presented in Table II. Body mass index was stable. AHI, Al and ODI were improved, only $\mathrm{AHI}$ and $\mathrm{Al}$ statistically significantly in both groups. Mean oxygen saturation was unchanged. Subjectively, ESS was significantly improved, while snoring VAS was improved but not significantly in both groups. The treatment success rate was $51.7 \%$ for the study group and $41.9 \%$

Table I. Preoperative comparison of the study and control group

\begin{tabular}{|lccc|}
\hline Parameter & $\begin{array}{c}\text { Study group } \\
\text { UPPP + RFBT }\end{array}$ & $\begin{array}{c}\text { Control group } \\
\text { UPPP }\end{array}$ & $\begin{array}{c}\text { Value } \\
\text { of } p\end{array}$ \\
\hline Age & $50.5 \pm 9.1$ & $47.9 \pm 8.7$ & NS \\
\hline Male/female & $62 / 17$ & $29 / 6$ & NS \\
\hline $\mathrm{BMI}$ & $28.1 \pm 3.1$ & $28.0 \pm 3.3$ & NS \\
\hline $\mathrm{AHI}$ & $28.7 \pm 17.1$ & $27.9 \pm 14.1$ & NS \\
\hline $\mathrm{Al}$ & $12.1 \pm 6.8$ & $11.9 \pm 5.7$ & NS \\
\hline ODI & $15.1 \pm 8.2$ & $15.3 \pm 6.3$ & NS \\
\hline Mean $\mathrm{SpO}_{2}[\%]$ & $95.4 \pm 3.9$ & $95.8 \pm 1.9$ & NS \\
\hline ESS & $10.6 \pm 3.8$ & $10.1 \pm 3.7$ & NS \\
\hline VAS snoring & $8.4 \pm 1.9$ & $8.3 \pm 2.1$ & NS \\
\hline
\end{tabular}

$B M I$ - body mass index, $A H I$ - apnea-hypopnea index, ODI - oxygen desaturation index, mean $\mathrm{SpO}_{2}$ - mean oxygen saturation, ESS Epworth Sleepiness Scale, VAS - visual analogue scale, NS - not statistically significant at $p<0.05$

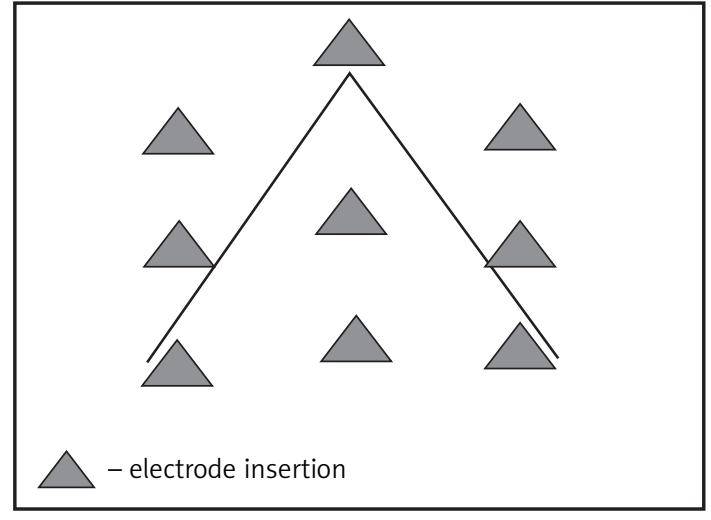

Figure 1. Scheme of RFBT application around the vallate papillae in the base of the tongue

for the control group, with a significant difference $(p=0.04)$.

No severe complications such as infections, abscesses or airway obstruction occurred. Two patients had postoperative bleeding from the tonsillar bed after UPPP, necessitating cauterization, both in the study group. Twenty-five patients (17 in the study group, 8 in the control group) suffered temporarily from velopharyngeal insufficiency after UPPP that resolved spontaneously within 2 weeks in all cases. Four patients had ulceration of the base of the tongue after RFBT with spontaneous cure within one week without any subjective adverse sensation. One patient after RFBT had a taste change in half of the tongue that resolved within 2 months.

Table II. Preoperative and postoperative values for the study and control group

\begin{tabular}{|c|c|c|c|}
\hline Parameter & $\begin{array}{c}\text { Preoperative } \\
\text { value }\end{array}$ & $\begin{array}{c}\text { Postoperative } \\
\text { value }\end{array}$ & $\begin{array}{l}\text { Value } \\
\text { of } p\end{array}$ \\
\hline \multicolumn{4}{|l|}{ Study group } \\
\hline $\mathrm{BMI}\left[\mathrm{kg} / \mathrm{m}^{2}\right]$ & $28.1 \pm 3.1$ & $28.3 \pm 3.5$ & NS \\
\hline $\mathrm{AHI}$ & $28.7 \pm 17.1$ & $14.1 \pm 18.2$ & 0.007 \\
\hline $\mathrm{Al}$ & $12.1 \pm 6.8$ & $5.9 \pm 4.3$ & 0.005 \\
\hline ODI & $15.1 \pm 8.2$ & $10.3 \pm 7.9$ & NS \\
\hline Mean $\mathrm{SpO}_{2}[\%]$ & $95.4 \pm 3.9$ & $95.3 \pm 2.8$ & NS \\
\hline ESS & $10.6 \pm 3.8$ & $7.3 \pm 3.2$ & 0.03 \\
\hline VAS snoring & $8.4 \pm 1.9$ & $6.0 \pm 3.1$ & NS \\
\hline \multicolumn{4}{|l|}{ Control group } \\
\hline $\mathrm{BMI}$ & $28.0 \pm 3.3$ & $28.3 \pm 3.0$ & NS \\
\hline $\mathrm{AHI}$ & $27.9 \pm 14.1$ & $18.1 \pm 10.5$ & 0.01 \\
\hline Al & $11.9 \pm 5.7$ & $8.2 \pm 4.1$ & 0.04 \\
\hline ODI & $15.3 \pm 6.3$ & $11.3 \pm 5.7$ & NS \\
\hline Mean $\mathrm{SpO}_{2}(\%)$ & $95.8 \pm 1.9$ & $95.9 \pm 2.5$ & NS \\
\hline ESS & $10.1 \pm 3.7$ & $7.1 \pm 3.9$ & 0.04 \\
\hline VAS snoring & $8.3 \pm 2.1$ & $6.3 \pm 3.6$ & NS \\
\hline
\end{tabular}




\section{Discussion}

Bipolar radiofrequency surgery is performed in different parts of the human body with diverse effects. Although volume reduction impact has been generally proposed it is not the result of treatment in each tissue type. Relevant changes after RFBT for obstructive sleep apnea could be observed neither for tongue volume or dimension nor for retrolingual space [9]. The effect of radiofrequency surgery of the tongue base may more likely be a result of change in upper airway collapsibility after scarring - postnecrotic fibrosis.

In most studies published on radiofrequency surgery of the base of the tongue for OSA several sessions with fewer lesions per session were performed $[2,5,7,10-12]$. Only one study described single-session radiofrequency surgery of the base of the tongue combined with UPPP for OSA [6]. Our study reports the highest number of patients treated by UPPP and bipolar RFBT in a single session and is the first one with a control group.

Bipolar RFBT was used in our study. An exactly defined volume of treated tissue is very important for safe preservation of neural and vascular supply that could be damaged if the lesion is too large. The bipolar arrangements of the electrode and the autostop function facilitate the power input to be best suited to the desired treatment and eliminate the above-mentioned risks. Current bipolar radiofrequency devices such as the CelonLab ENT unit significantly reduce application time compared to previously used ones, especially compared to monopolar ones. As the average duration of application is $8 \mathrm{~s}$ per puncture, the whole procedure time is less than 5 min for our 9-puncture schedule counting all the procedure including the tongue base access, proper radiofrequency application and final operation field control. Radiofrequency thermotherapy of the base of the tongue combined with UPPP as a single session procedure had in our study an average operation time of 55 min, so it added 10\% more time compared to the solely UPPP procedure.

Bipolar RFBT is a minimally invasive and safe procedure that is easy to perform and well tolerated. The majority of complications in our study were UPPP-related: two post-tonsillectomy bleeding events and 25 cases of transient velopharyngeal insufficiency. From RFBT-related complications we reported four ulcerations of the base of the tongue and one taste change in half of the tongue. These lesions healed spontaneously without sequelae. Ulcerations developed as a result of a too superficial positioning of the electrode probe below the mucosa. For prevention of injury of the lingual neurovascular bundle the applicator is covered by a synthetic coat that protects the applicator from being inserted too far in the tissue. Moreover, the surgeon must avoid too lateral application of the probe. In studies using bipolar RFBT Herder et al. [13] reported two cases of transient and one of permanent hypoglossal nerve paresis, Eun et al. [6] reported one tongue base ulceration and one transient taste change, while Broek et al. [5] reported two cases of transient lingual and hypoglossal paresis, and one edema of the tongue.

The overall success rate of combined bipolar RFBT and UPPP has been recently reported as 49\% and $53.6 \%$, respectively $[5,6]$. Our result of $51.7 \%$ is comparable. Our study consists of the highest number of patients that suffered from a more severe level of OSA. The pre-treatment value of $\mathrm{AHI}$ was higher in our study group - 28.7, in comparison to 17.8 and 22.9 , respectively $[5,6]$. In our study the overall success rate increased from 41.9 for UPPP alone to $51.7 \%$ for UPPP with RFBT.

Our study has some limitations. It was a retrospective study and even though it is the largest published group the number of subjects is limited. The ideal study for the exact evaluation of the effectiveness of any treatment would be a prospective, randomized trial with the control group treated by placebo. As was shown by Stuck, it is feasible in radiofrequency surgery of the soft palate for snoring but it would not be possible for such invasive surgery as UPPP [14]. Another possibility is the control group treated by the gold standard in the treatment of OSA - continuous positive airway pressure (CPAP). All patients included in our study refused or did not tolerate CPAP. We used as the control group patients treated with UPPP alone, i.e. the most frequently used standard surgery for OSA. We proved improvement of the effectiveness by adding RFBT to UPPP in patients with both oropharyngeal and hypopharyngeal obstruction.

Although respiratory parameters such as $\mathrm{AHI}$ and ODI are widely used for evaluation of the effectiveness of the treatment for OSA, the most important health parameter is the influence on cardiovascular morbidity and mortality $[15,16]$. Long-term results for combined bipolar RFBT and UPPP are not yet accessible since the method has been used for a relatively short time with respect to assessment of cardiovascular morbidity and mortality.

In conclusion, combined bipolar radiofrequency surgery of the base of the tongue and uvulopalatopharyngoplasty as a single-session procedure is a minimally invasive, well-tolerated and safe method in the treatment of OSA. It is effective in reducing respiratory parameters and improving subjective symptoms of OSA. The advantages of this method are the single session procedure, simple feasibility, bipolar technique and short time of the procedure. Long-term follow-up should be completed to better evaluate long-term results and to estimate the impact on cardiovascular morbidity of treated patients. 


\section{Acknowledgments}

This work was supported by the Internal Grant Agency of MZCR NT 13488, PRVOUK 27-1 and SVV 266513.

\section{References}

1. Fujita S, Conway W, Zorick F, Roth T. Surgical correction of anatomic abnormalities in obstructive sleep apnea syndrome: uvulopalatopharyngoplasty. Otolaryngol Head Neck Surg 1981; 89: 923-34.

2. Powel NB, Riley RW, Guilleminault C. Radiofrequency tongue base reduction in sleep disordered breathing: pilot study. Otolaryngol Head Neck Surg 1999; 120: 656-64.

3. Farrar J, Ryan J, Oliver E, Gillespie M. Radiofrequency ablation for the treatment of obstructive sleep apnea: a meta-analysis. Laryngoscope 2008; 118: 1878-83.

4. Neruntarat C, Chantapant S. Radiofrequency surgery for the treatment of obstructive sleep apnea: short-term and long-term results. Otolaryngol Head Neck Surg 2009; 141: 722-6.

5. Broek E, Richard W, Tinteren H, de Vries N. UPPP combined with radiofrequency thermotherapy of the tongue base for the treatment of obstructive sleep apnea syndrome. Eur Arch Otorhinolaryngol 2008; 265: 1361-5.

6. Eun YG, Kim SW, Kwon KH, Byun JY, Lee KH. Singlesession radiofrequency tongue base reduction combined with uvulopalatopharyngoplasty for obstructive sleep apnea syndrome. Eur Arch Otorhinolaryngol 2008; 265: 1495-500.

7. Nelson LM. Combined temperature-controlled radiofrequency tongue reduction and UPPP in apnea surgery. Ear Nose Throat J 2001; 80: 640-4.

8. Friedman M, Ibrahim H, Joseph NJ. Staging of obstructive sleep apnea/hypopnea syndrome: a guide to appropriate treatment. Laryngoscope 2004; 114: 454-9.

9. Stuck BA, Köpke J, Hörmann K, et al. Volumetric tissue reduction in radiofrequency surgery of the tongue base. Otolaryngol Head Neck Surg 2005; 132: 132-5.

10. Friedman M, Ibrahim H, Lee G, Joseph NJ. Combined uvulopalatopharyngoplasty and radiofrequency tongue base reduction for treatment of obstructive sleep apnea/ hypopnea syndrome. Otolaryngol Head Neck Surg 2003; 129: 611-21.

11. Jacobowitz O. Palatal and tongue base surgery for surgical treatment of obstructive sleep apnea: a prospective study. Otolaryngol Head Neck Surg 2006; 135: 258-64.

12. Steward DL. Effectiveness of multilevel (tongue and palate) radiofrequency tissue ablation for patients with obstructive sleep apnea syndrome. Laryngoscope 2004; 114: 2073-84.

13. Herder C, Kox D, Tinteren H, de Vries N. Bipolar radiofrequency induced thermotherapy of the tongue base: its complication, acceptance and effectiveness under local anesthesia. Eur Arch Otorhinolaryngol 2006; 263: 1031-40.

14. Stuck BA, Sauter A, Hörmann K, Verse T, Maurer JT. Radiofrequency surgery of the soft palate in the treatment of snoring. A placebo-controlled trial. Sleep 2005; 28: 847-50.

15. Stuck BA. A critical evaluation of surgery for sleep disordered breathing. In: Hörmann K (ed.). An update on sleep disordered breathing. Rheinware Verlag, Möchengladbach 2006; 75-92.

16. Korostovtseva L, Sviryaev Y, Zvartau N, Konradi A, Kalinkin A. Prognosis and cardiovascular morbidity and mortality in prospective study of hypertensive patients with obstructive sleep apnea syndrome in St Petersburg, Russia. Med Sci Monit 2011; 17: CR146-153. 